\title{
Communication Strategies in the Written Medium: The Effect of Language Proficiency*
}

\author{
Mohammad Aliakbari (Ilam)/Nabi Karimi Allvar (Tehran)
}

\begin{abstract}
Although much has been written on 'communication strategies' (CS), in the sense of potentially conscious plans for solving problems encountered in reaching particular communicative goals, there still remain large gaps in our knowledge in this respect. One area worthy of attention, and not totally clarified, is whether these strategies are applied and workable in writing as frequently as they are in speaking tasks. Accordingly, the present study aimed at pitting the use of CS against language proficiency level in argumentative writing of Iranian university students. The results of the Chi-Square tests run for the purpose of investigating the hypotheses showed that language proficiency was highly related with the use of more Reconceptualization Strategies which are thought to be psycho linguistically more demanding compared with Substitution Strategies.
\end{abstract}

\section{$1 \quad$ Introduction}

It is a solid fact that no second language learner's, or even no native speaker's linguistic repertoire or control of language is perfect. Native as well as non-native speakers of a particular language sometimes struggle to find the appropriate expression or grammatical structure when they intend to get their meaning across. The ways in which an individual speaker attempts to compensate for this gap between what he intends to communicate and his immediately available linguistic resources are known as communication strategies CS (Faucette 2001). They are in fact "conceptualized as problem-solving devices whose conscious implementation is directed towards counteracting the imbalance between ends and means typical of L2 learners' productive uses of language" (Manchon 2000: 14f.). The term CS was first coined and introduced by Selinker (1972) in his seminal article titled "interlangauge". They have, since then, been identified as one of the key areas of investigation within Second Language Acquisition research and thus have been extensively investigated in research on second language learning and teaching. But the bulk of theoretical and empirical studies in the field have centered on the CS deployed by L2 users in oral production tasks of second language learners (Manchon 2000) and it has not attracted much attention to itself in the written medium. However, it is often theoretically claimed that CSs can have as many uses and repercussions in the written medium as in the spoken medium with which CSs are often identified. This study, thus, is an attempt to empirically investigate the use of CSs in the written medium by Iranian second language learners. To set the ground for the actual problem the study is to tap into, a sketchy account of the literature of the topic is provided.

\footnotetext{
* The authors would like to thank the two anonymous reviewers for their helpful comments on a previous draft of this manuscript.
} 


\section{Review of the Related Theory and Literature}

\subsection{Communication Strategies in the Written Medium}

As was pointed out before, the concept of communication strategy in writing is quite a Cinderella subject and has not gotten a proper deal. Very few studies have directly touched upon the concept of CS in writing, but CSs have gotten some coverage under some other names such as error analysis, writing strategy, dictionary use, etc. For instance there are often indications in the literature for distinguishing three error categories, known more specifically as 'slips', 'errors' and 'attempts'. The third of these are where students have no real idea how to structure what they want to mean, and are characterized by typically being correctable neither by the learner himself/herself (as slips are) nor by the learners' peers (as errors are). They are beyond the learner and peers' competence and correctable only by a teacher. They are in fact nothing other than errors arising from use of CS (in the traditionally central meaning of this term), under another name (Edge 1989, cited in Keyvani 2000).

Another research area that touches upon written CS is general writing strategy research. Here, however, usually strategies of the CS type are lost in the general classification system used for writing behaviors or strategies by individual researchers, and are usually overshadowed by other terms and concepts. These classifications at present remain often rather general. In the well-known Flower and Hayes (1981) model of the writing process, CS could probably occur in any of the three main categories of processing - either in 'planning', 'translating' (which in this scheme means turning thought into written form) or 'reviewing'. When planning, for example, one may look ahead to how one would express what one plans to say, decide one's linguistic resources are not up to the task, and change the intended message accordingly (with some 'avoidance'). When 'translating' (i.e. actually writing draft text) one may resolve problems of linguistic expression e.g. by paraphrasing or, through word processing, using an online dictionary, or perhaps leave a gap and later on, when reviewing, one may stop and ask someone which is the same appeal to authority that is a prominent CS. We may contrast the lack of discussion of written CS in relation to such models with the lively discussion in the oral CS literature. Oral CSs were first interpreted in terms of a model of speaking by Færch and Kasper (1983) and subsequently the speaking models of Levelt (1989) and others that have been invoked (e.g. by Poulisse 1990). Also in a study done by Whalen and Ménard (1995), which can be considered as an example of a good recent study on L2 writing strategies, we find several references to what are in point of fact CS (e.g. circumlocution, dictionary use). However, they are not named as CS or distinguished in terms of their function. Recently, however, the direct experimental discussion of CS in writing has been started. A good case in point is a study done by Ambrose (1998), which deals with communication strategies used in the writing of answers in Biology by ESL first year BSc students of the University of Botswana. The researcher examined the four macro strategies used by the subjects: risk taking, risk avoidance, L2 based strategies and semantic simplification. The results showed that while many ESL students preferred to use L2 based strategies such as circumlocution, generalisation and paraphrase, these strategies did not help the students in their performance to a whole extent because of the restrictive nature of scientific genre which requires the use of specific and precise registers for a given writing task. The study also demonstrated that those students who were tending to take risks by exploiting their resource expansion strategies, irrespective of the correctness of their grammatical constructions, did better while those students who opted for semantic simplification and risk avoidance performed far less well. The study also concluded that the overall writing proficiency of the L2 learners could be greatly enhanced if TEFL practitioners paid greater attention to those tasks and activities that enhance their strategic competence. 


\subsection{Taxonomies of Communication Strategies}

Ever since Selinker's (1972) article on interlanguage introduced the notion of second language communication strategies, various researchers have gone about classifying these strategies in various ways. From among these classifications, one can allude to Váradi (1973) and Tarone (1977) who introduced a classification of communication strategies that were used in subsequent research. Tarone (1977) described her taxonomy as a system which provides the best tool to make sense of the behavior of the subjects in communicative situations. The author herself pointed out the lack of generality of her taxonomy. However, other researchers have adapted Tarone's (1977) typology and introduced other strategies (Paribakht 1985). This fact has promoted the existence of a rather confusing multitude of different strategies of ambiguous validity. Bialystok (1990) believes that the variety of taxonomies proposed in the literature differ primarily in terminology and overall categorizing principles rather than in the substance of specific strategies. The following represents taxonomy of communication strategies based on the above stated conceptualizations and mainly developed out of Tarone (1977) classification.

\section{A. Avoidance or Reduction Strategies}

1. Message abandonment (leaving a message unfinished because of linguistic difficulties)

2. Topic avoidance (avoiding topic areas or concepts which pose linguistic difficulties)

B. Achievement or Compensatory Strategies

3. Circumlocution (describing or exemplifying the target object or action)

4. Approximation (using an alternative term which expresses the meaning of the target lexical items as closely as possible (e.g. ship for sail boat)

5. Use of all-purpose words (extending a general empty lexical item to contexts where specific words are lacking (e.g. overuse of thing)

6. Word-coinage (creating a non-existing L2 word based on a supposed rule)

7. Use of nonlinguistic means (mime, gesture, facial expression)

8. Literal translation (translating literally a lexical item an idiom, a compound word or structure from L1 to L2)

9. Foreignizing (using a L1 word by adjusting it to L2 phonologically)

10. Code switching (using a L1 word with L1 pronunciation)

11. Appeal for help (turning to the conversation partner for help, directly or indirectly)

C. Time Gaining Strategies

12. Use of fillers/hesitation (Devices using filling words or gambits to fill in pauses and to gain time to think)

In the late 1980s, some researchers at Nijmegen University (Kellerman et al. 1987) criticized the existing typologies of communication strategies as mainly product-oriented, since these tended to show strategy types with isolated examples, rather than represent how these typologies could be applied to cohesive speech or writing. They believed that these typologies were merely descriptive since they focused only on the linguistic product.

The Nijmegen University Group found two major deficits in product-oriented taxonomies of communication strategies. These deficits were: a failure to distinguish the psychological process from the linguistic product, as well as to consider the linguistic and non-linguistic constraints that influence the choice of a particular strategy. 
For the above-mentioned reasons, this group of researchers proposed an alternative taxonomy of communication strategies, which is based on the assumption that identifying the cognitive processes which underlie the choice of a strategy as well as taking into account the factors involved in such selection is essential:

I. Conceptual archistrategy (manipulating the target concept to make it expressible through available linguistic resources)

1. A. Analytic Strategies (specifying characteristic features of the concept (e.g. circumlocution)

1. B. Holistic Strategies (using a different concept which shares characteristics with the target item (e.g. approximation)

II. Linguistic/CodeArchistrategy (manipulating the speakers' linguistic knowledge)

2. A. Morphological Creativity (creating a new word by applying L2 morphological rules to an L2 word (e.g. grammatical word coinage)

2. B. Transfer (from another language)

Poulisse (1993) criticizes the above quoted typology by arguing that the distinction between conceptual and linguistic strategies does not refer to different processes involved in the production of these strategies. According to Poulisse, both conceptual strategies of the holistic type and the linguistic strategy of transfer refer to the same process, namely that of substitution, regardless of whether the word being replaced belongs to a same or a different language.

Apart from this, Poulisse (1993) points to great differences in the processes underlying analytic and holistic conceptual strategies. In the author's view, analytic strategies (e.g. circumlocution) require the planning and execution of new messages, which involves creating new syntactic plans, and selecting new lexical items from the mental lexicon. On the other hand, holistic strategies (e.g. approximation) require the addition or replacement of some of the conceptual features in the concept which the speaker is about to produce. Bearing this difference in mind, and considering the processes involved in the use of linguistic transfer strategies, Poulisse (1993) states that holistic strategies seem to be more similar to linguistic transfer strategies than to conceptual analytic ones.

Poulisse, thus, presents an alternative typology of communication strategies. This taxonomy fulfills all the criteria, which, in the author's opinion, any theory of communication strategies use should meet. These criteria are the following:

It should allow one to define CS use and to operationalize the distinction between strategic and nonstrategic language use; it should capture the differences between the CS types that have been distinguished in a theoretically satisfying manner; and it should enable one to explain empirical findings that have been obtained to date and to predict future findings.

The typology of communication strategies suggested by Poulisse (1993) is as follows:

A. Substitution (substitution of one lexical item for another, whether it be an L1 for L2 item).

B. Substitution plus type (substitutions which require phonological and/or morphological adaptation before they are articulated e.g. foreignizing, morphological creativity).

C. Reconceptualization (a change in the preverbal message which involves more than a single chunk, (e.g. paraphrase).

The above taxonomy has been developed in accordance with Levelt's (1989) model of speech production, which includes the following parts. This taxonomy is what we have used in this study and the reason for its adoption is its obvious merits compared with the other ones 
reviewed here, though we have deleted the second ramification as it is more related to oral production.

\section{$3 \quad$ Statement of the Problem}

Although the relevance of the concept of CS with written production has been well affirmed theoretically, very few studies have been devoted to the empirical investigation this concept in the written medium.

There indeed remains the problem of how the use of these strategies relates to variables usually associated with them, in the written medium as opposed to the spoken medium. Thus, the present study sets out to study CSs in terms of their relation to language proficiency and their manifestation in the written production of Iranian university students. The rationale behind this study is to take a step towards the empirical investigation of CSs in the written medium which has been a neglected subject for so long a time and to attempt to bring it into the focus of attention. The study will, moreover, shed some light on the whole construct of communication strategy as one of the integral but still nebulous aspects of communicative competence.

\subsection{Research Question}

In order to meet the above-stated gaps, the study specifically deals with the following research question:

Does language proficiency exert a significant effect on the types of communication strategies employed by Iranian English language learners in their written productions?

\subsection{Research Hypothesis}

With reference to the statement of the problem and the questions of the study this null hypothesis has been formed.

Language proficiency does not exert a significant effect on the types of communication strategies employed by Iranian English language learners in their written productions?

\section{$4 \quad$ Method}

\subsection{Participants}

The participants of the study included 30 students of English language and Literature, selected from among 57 students at Ilam University based on their performance on TOEFL-like proficiency test, a modified version of TOEFL mostly conducted in Iranian contexts as an entrance test for various cross-country educational programs with a reasonable degree of reliability. It consisted of 40 grammar tests, 40 vocabulary test and 20 reading comprehension tests. The subjects were 12 Male and 18 Female students, studying at third and fourth semesters. The mean score of the high-proficiency group was 60 on the above-described test and the mean score of the low-proficiency group was 35. Their age ranged from 20 to 26.

\subsection{Procedure}

As a first step, 57 students majoring in English literature at Ilam University were given a screening TOEFL-like test. The top fifteen students who scored the highest were selected as the high-proficient group and the bottom fifteen students with the lowest scores were selected as the low-proficient group. 
Since the study aimed at identifying CSs employed by EFL learners in their written performance as a function of their differential proficiency level, both high and low-proficient groups were given 45 minutes to write an essay of about 150 words arguing on the assumption that 'people can be happy without money' while thinking aloud and writing their mind in a separate piece of paper. The topic was chosen because it was of a general and controversial nature and also because the aim was to come up with a sample of argumentative writing piece. The subjects were also interviewed to retrospect into the process they had been following in their writing.

\subsection{Classification Scheme}

The classification of communication strategies was based on Poulisse (1993) which proposes two main categories for communication strategies: Substitution Strategies (SS) and Reconceptualization Strategies (RS). In adopting SS, a speaker may replace an intended word with one word (e.g., "animal" for DOG, or "table" for CHAIR) while with RS, a speaker uses more than one word to express an intended word (e.g., "wave receiver" for AERIAL). Poulisse (1993) further maintains that SS and RS have different psycholinguistic processes, and that RS is more demanding.

To identify and classify LCSs, Poulisse's (1993) decision tree, which has already been used in many studies, was used. The decision tree contains 7 questions: (1) Is there a problem indicator? (2) Is it of a lexical nature? (3) Is the problem solved immediately? (4) Does the participant try to express the meaning of the word? (5) Is there a strategy? (6) Does the speaker use more than two words to express the intended word? (7) Is the substituted word correct in L2?

It deserves notice that in identification tree adopted, answers to questions in oral production are relatively easier than in written production. This is because in the former the researcher can actually observe if there is a problem. $\mathrm{S} / \mathrm{he}$ can also reorganize its type if there is any. But when it comes to writing the researcher cannot read the participants' minds. As a result, to ensure the quality of the answers to these questions we used the participants' while-writing reflections and retrospective interviews were further designed. The data collected through these reflections and interviews, accompanied with the CS identification decision tree, were all considered in identifying and classifying the CSs based on Poulisse's (1993) taxonomy.

The subjects' compositions were collected and their strategies were identified by one of the researchers and a colleague whose identification system was reported to be around $84 \%$ which was strong enough to establish creditability of the identification scheme in this study. Bellow comes a few examples of the strategies identified from students' writings.

\section{Examples of Substitution Strategy:}

He made a shart (Persian word, meaning stipulation) if his son ...

(The participant simply uses the native language item without brothering to translate.)

He washed and rendered his habitual deed.

(Habitual deed is used as a substitute for religious service).

Examples of Reconceptualization Strategy:

They often go to the computer which gives money.

(The participant wants to say "Automatic Teller Machine (ATM)", he has defined it indeed.)

Most of them live in houses which have a place for swimming ...

(The participant intends to say "pool".) 


\subsection{Data Collection}

As noted before, throughout the investigations, different types of data were collected from the subjects. In the first place, argumentative essays were collected from both of high and low proficiency groups. Students were also asked to reflect on their thought processes in the course of their writing mainly in terms of focus on lexical difficulties they encountered and the ways they went about obviating those problems (Appendix 1). The main purpose behind these reflections was to help us in identifying the communication strategies used in the participants' writings. It is worthy of mentioning that the participants received two sessions of instructions about how to carry out while-writing reflections and retrospection into their thought processes during their writing. Moreover, an informal interview process was followed with each of the participants as to the processes they have been through upon trying to get their meanings across in their written productions and the reasons behind adopting particular ways and strategies in communicating their intent across.

\section{$5 \quad$ Data Analysis}

To test the first hypothesis of the study, a two-way Chi-square test was run the procedure of which is described below. Table 1 represents the schematic representation and the frequency of the strategies identified.

\begin{tabular}{|c|c|c|}
\hline & High-Proficient & Low-Proficient \\
\hline RS & 27 & 14 \\
\hline SS & 15 & 38 \\
\hline
\end{tabular}

Table 1: Schematic representation and the frequency of the strategies

\begin{tabular}{|c|c|c|c|c|c|}
\hline & \multicolumn{2}{|c|}{ Proficiency } & \multirow[b]{2}{*}{ Total } \\
\hline & & & $\begin{array}{c}\text { High- } \\
\text { Proficient }\end{array}$ & $\begin{array}{c}\text { Low- } \\
\text { Proficient }\end{array}$ & \\
\hline \multirow[t]{2}{*}{ CS Type } & $\mathrm{RS}$ & $\begin{array}{l}\text { Count } \\
\text { Expected Count }\end{array}$ & $\begin{array}{r}27 \\
18.3\end{array}$ & $\begin{array}{r}14 \\
22.7\end{array}$ & $\begin{array}{r}41 \\
41.0\end{array}$ \\
\hline & SS & $\begin{array}{l}\text { Count } \\
\text { Expected Count }\end{array}$ & $\begin{array}{r}15 \\
23.7\end{array}$ & $\begin{array}{r}28 \\
29.3\end{array}$ & $\begin{array}{r}53 \\
53.0\end{array}$ \\
\hline \multicolumn{2}{|l|}{ Total } & $\begin{array}{l}\text { Count } \\
\text { Expected Count }\end{array}$ & $\begin{array}{r}42 \\
42.0\end{array}$ & $\begin{array}{r}52 \\
52.0\end{array}$ & $\begin{array}{r}94 \\
94.0\end{array}$ \\
\hline
\end{tabular}

Table 2: CS and Proficiency Cross tabulation

A Chi-square test was run for the purpose of the investigating this hypothesis, the results are as follows:

\begin{tabular}{|c|c|c|c|c|c|}
\hline & Value & $\mathrm{df}$ & $\begin{array}{c}\text { Asymp. Sig. } \\
\text { (2-sided) }\end{array}$ & $\begin{array}{l}\text { Exact Sig. } \\
\text { (2-sided) }\end{array}$ & $\begin{array}{l}\text { Exact Sig. } \\
\text { (1-sided) }\end{array}$ \\
\hline Pearson Chi-Square & $13.189^{\mathrm{b}}$ & 1 & .000 & & \\
\hline Continuity Correction ${ }^{\mathrm{a}}$ & 11.713 & 1 & .001 & & \\
\hline Likelihood Ratio & 13.449 & 1 & .000 & & \\
\hline Fisher's Exact Test & & & & .000 & .000 \\
\hline Linear-by-Linear Association & 13.048 & 1 & .000 & & \\
\hline $\mathrm{N}$ of Valid Cases & 94 & & & & \\
\hline
\end{tabular}

Table 3: Chi-Square Tests.

${ }^{a}$ Computed only for a $2 \times 2$ table.

${ }^{b} 0$ cells $(.0 \%)$ have expected count less than 5 . The minimum expected count is 18.32 . 
As one can see from the Chi-square tests table, $\left(\chi^{2}=13.18, \mathrm{df}=1 ; \mathrm{p}<0.05\right)$, we can feel quite confident in rejecting the null hypothesis and thus, it can be concluded that there is a significant association and relationship between language proficiency and the types of communication strategies employed by second language EFL learners in the written medium.

Besides these quantitative analyses, the qualitative data gathered and analyzed including while-writing introspective reflections, together with the identification decision tree, helped greatly in identifying and classifying CSs. Through the while-writing reflections, the learners were asked to write the difficulties of lexical nature and the ways they went about their obviation. These data provided answers to the questions put forward in the identification decision tree. The analyses of the retrospective interview yielded roughly the same results arrived at through the while-writing reflections and the quantitative analyses. It lent even more support to the tendency towards more use of RS and less use of SS as a function of the increase in language proficiency. Many of the participants also attested to the more usefulness of the strategies of RS type in that they thought these strategies help them better in clearly articulating their intent. In their interviews, the lower-proficiency participants demonstrated a preference for substitution strategies. They cited more ease and less competence in roundabout explanations as the reasons why they preferred substitution strategies over reconceptualization ones.

\section{Discussion and Conclusion}

The quantitative analyses suggest that with higher language proficiency the rate of Reconceptualization Strategies used by each participant increased and the rate of Substitution Strategies decreased. In other words, the group with higher language proficiency used significantly more RS than the other group with a lower level of language proficiency. The justification for the above results can be inferred from the qualitative analyses of the retrospective interviews. The qualitative analyses of these interviews showed that the participants regarded RS as more useful than SS in that they believed RS could specify the intended concepts more clearly than SS. This is in line with Poulisse (1993) in that it maintains that from a processing point of view, RS is psychologically more demanding than SS. Thus, it can be thought that learners with sufficient language proficiency produce lexical items more easily and that they have acquired many words and are able to use them more fluently. This fluent processing of lexical items reduces processing load of speech production and allows them to use more RSs. In contrast, RS is too demanding for learners with insufficient language knowledge, for the overall processing demands of speech production are too high.

The study further reveals that uses of lexical communication strategies are different between learners with a high proficiency and those with a low proficiency and that learners with a high proficiency tend to use psychologically more demanding communication strategies. This may be due to the fact that such demanding communication strategies are unfeasible for learners with a low proficiency because they have to concentrate on the process of speech production itself. The study also implies that an increase in language proficiency may make verification mechanism more sensitive in lexical retrieval.

The study also reveals that an increase in language proficiency may result in a considerable reduction in the use of strategies. This seems natural because with higher proficiency, the learners' linguistic repertoire becomes more established and more developed which reduces the gaps in their performance to fill with strategies.

A final important conclusion which can be inferred from the results is that teaching communication strategies to lower-proficiency students can enormously enhance their performance. In so doing, special stress should be put on teaching reconceptualization strategies with which 
the learners seem to have difficulties and due to the fact that they are more useful than substitution strategies.

\section{References}

Ambrose, B. Chimbganda (1998): "Communication and Study Skills Unit". Journal of Language Learning 32/2.

Bialystok, Ellen (1990): Communication Strategies. A Psychological Analysis of Second Language Use. Oxford.

Bialystok, Ellen/Fröhlich, Maria (1980): "Oral communication strategies for lexical difficulties". Interlangauge Studies Bulletin 5: 3-30.

Dechert, Hans-Wilhelm (1983): "How a story is done in second language". In: Faérch, Claus/ Kasper, Gabriele (1983): Strategies in Interlanguage Communication. London.

Dornyei, Zoltan/Scott, Mary Lee (1997): "Communication strategies in a second language. Definitions and Taxonomies". Language Learning 47/1: 173-210.

Faérch, Claus/Kasper, Gabriele (1983): Strategies in Interlanguage Communication. London.

Faucette, Priscilla (2001): "A pedagogical perspective on communication strategies. Benefits of training and an analysis of English language teaching materials". Second Language Studies 19/2: 1-40.

Flymen, Anna (1997): "Communication Strategies in French as a Foreign Language". Lund University Working Papers 46: 57-73.

Haastrup, Kirsten/Philipson, Robert (1983): "Achievement Strategies in Learner/Native Speaker Interaction". In: Faérch, Claus/Kasper, Gabriele (1983): Strategies in Interlanguage Communication. London.

Flower, Linda M./Hayes, John R. (1981): "A cognitive process theory of writing". College Composition and Communication 32: 365-387.

Karimi Alvar, Nabi (2003): The relationship between elicitation task nature and Iranian EFL students' use of oral communication strategies. Unpublished Master Thesis, Tarbiat Modaress University, Tehran.

Kasper, Gabriele/Kellerman, Eric (1997): Communication Strategies. London.

Kellerman, Eric/Bongaerts, Theo/Poulisse, Nanda (1987): "Strategy and system in L2 referential communication". In: Ellis, Rod (ed.): Second Language Acquisition in Context. Englewood Cliffs, New Jersey.

Keyvani, Azam (2000): The Relationship between Extroversion/Introversion and Communication Strategies in Speaking. Unpublished Master Thesis, Alzahra University, Tehran.

Levelt, Willem J. M. (1989): Speaking. Cambridge, MA.

Manchón, Rosa María (2000): "Fostering the autonomous use of communication strategies in the foreign language classroom". Links \& Letters 7: 13-27.

Paribakht, Tahereh (1985): "Strategic Competence and Language Proficiency". Applied Linguistics 6/2: 132-146.

Poulisse, Nanda (1990): The Use of Compensatory Strategies by Dutch Learners of English. Berlin.

Poulisse, Nanda/Schils, Erik (1989): "The influence of task- and proficiency-related factors on the use of compensatory strategies. A quantitative analysis". Language Learning 39/1: $15-48$.

Rossiter, Marian J. (2003): "'It's like chicken but bigger'. Effects of communication strategy in the ESL Classroom". Canadian Modern Language Review 60: 105-121.

Selinker, Larry (1972): "Interlanguage". International Review Applied Linguistics (IRAL) 10/3: 209-231.

Stern, Hans Heinrich (1991): Fundamental Concepts of Language Teaching. Oxford. 
Tarone, Elaine (1977): "Conscious communication strategies in interlanguage. A progress report". In: Brown, Henry Douglas et al. (eds.): On TESOL '77. Teaching and Learning English as a Second Language. Washington, D.C.

Váradi, Tamas (1973): "Strategies of target langage learner communication. Message adjustment". Paper presented at the Sixth Conference of the Romanian-English Linguistics Project in Timisoara.

Wagner, Johannes (1983): "Dann du tagen eineeeee - weisse platte - an analysis of Interlanguage communications in instructions". In: Faérch, Claus/Kasper, Gabriele (1983): Strategies in Interlanguage Communication. London.

Whalen, Karen/Menard, Nathan (1985): "L1 and L2 Writers' Strategic and Linguistic Knowledge. A Model of Multiple-Level Discourse Processing". Language Learning 45/3: 381418.

Wongsawang, Piyasuda (2000): "Culture-Specific Notions in L2 Communication Strategies". Second Language Studies 19/2: 111-135.

\section{Appendix 1}

\section{While-Writing Reflection Worksheet}

\section{Student's Code:}

As you are writing on the assigned topics, write, on the left-hand side, the concepts which you think you have problem with in communicating and on the right-hand side, write the ways you choose to get the problematic concept across.

Problematic Concepts

The way(s) you ultimately choose to communicate them. 


\section{Appendix 2}

\section{The Retrospective Interview Questions}

\section{Student's Code:}

Question Number 1:

- What ways did you use to get across the lexical difficulties you encountered in your writing?

Question Number 2:

- Why did you use these ways to obviate lexical difficulties?

\section{Appendix 3}

An example student essay together with Lexical Communication Strategies written in bold and underlined.

\section{One can be happy without money.}

I think if we emphasize our life on love, life will be very attractive. Love is a connection that connects everyone in everywhere. Money is a tool for living that solve financial problems, but it's not a goal so without money there is happiness in inside.

Psychologists have received (this stems from Persian use, instead of found out) that what we are thinking is what we are and it's based on our thinkings so if we think we are happy without money, we will be.

There are many ways and reasons for being very happy without money. One of them is exercise. Exercise will release endorphin in our body that makes us happy.

Another is healthy. Noticing one healthy can help in being very happy and there is no or little need for money. The other can be noticing that God has given us very blessings. God has given us eyes that much better than from camera and brain, foot, hands. When one of us misses one of the members in our body, we pay attention to this blessing that before we didn't attention to them. When we miss them, we want give all of our money for reget (the student uses this word instead of regaining) that members. The last is helping to each other. For against (when asked the student what she meant by this phrase, she said she had intended to say "to tackle") problems we can help and understand each other. Co-thinking (a word used to convey "Consultation") is necessary and useful in solving problems so without money happiness is possible. 